\title{
Avaliação de escolas públicas através de desenhos: um estudo comparativo de três escolas da cidade do Rio de Janeiro
}

\author{
Edson de Souza Filho* \\ - Fernanda Insfrán*** \\ - Ana Paula Magalhães*** \\ Daise Rosas ${ }^{* * * * *}$
}

\section{Resumo}

0 objetivo deste trabalho foi testar um método de avaliação de escolas públicas através do desenho e refletir sobre sua aplicabilidade para compreensão de fenômenos psicossociais que ocorrem no ambiente escolar. Supomos que a linguagem verbal tende a favorecer as normas sociais mais influentes no ambiente acadêmico e fora dele, prejudicando a expressão de indivíduos e grupos que adotam outras normas, supostamente menos legitimas e/ou não reconhecidas socialmente. Os participantes foram estudantes de ensino fundamental e médio de escolas públicas na cidade do Rio de Janeiro. Eles fizeram um desenho da escola onde estudavam e, em seguida, outro desenho sobre o interior da mesma e, enfim, verbalizaram sobre os desenhos feitos. Constatamos tendências de valorização positiva da imagem da escola e menor envolvimento em atividades típicas de ensino e aprendizagem. A escola desenhada indicou necessidade de um trabalho de dinâmica grupal no ambiente escolar de modo a inserir os estudantes.

Palavras-chave: Avaliação escolar. Desenho da escola. Psicologia social da educação.

\footnotetext{
* Doutor em Psicologia Social, École des Hautes Études en Sciences Sociales. Paris, FR; Professor do Programa de Pós-graduação em Psicologia da Universidade Federal do Rio de Janeiro (UFRJ).

E-mail: edsouzafilho@gmail.com

** Doutora em Psicologia, UFRJ; Professora de Psicologia Educacional e Orientação Profissional da Universidade Santa Úrsula. E-mail: nandainsfran79@gmail.com

**** Mestre em Psicologia Social, UFRJ. E-mail: apsm8@ig.com.br

***** Doutoranda em Psicologia, UFRJ; Diretora Comercial da EOSS Consulting LLC. E-mail: daise@eossconsulting.com
} 


\section{Evaluation of public schools through drawings : a comparative study of three schools in the city of Rio de Janeiro Abstract}

This work aimed at testing a method of evaluation of public schools through drawings and to reflect on its applicability to the understanding of psychosocial phenomena taking place in the school's realm. We assume that verbal language tends to favor those social norms which are more influential in the academic milieu and away, which is detrimental to the expression of individuals and groups who adopt other norms thought to be less legitimate and/or socially unrecognized. The participants were elementary and high school students of public schools in the city of Rio de Janeiro. They made a drawing of the school in which they study at and, following, one indoor drawing of it and, finally, they described the drawings they had just made. We verified a tendency to value positively the school's image and less involvement in typical activities of teaching and learning. The drawings of the school pointed to the necessity of a group dynamic work in order to place the students in the school's realm.

Keywords: School's evaluation. Drawing of the school. Social psychology of education.

\section{Evaluación de escuelas públicas a través de dibujos - un estudio comparativo de tres escuelas de la ciudad de Rio de Janeiro Resumen}

El objetivo de este trabajo fue testar un método de evaluación de escuelas públicas a través del dibujo y reflexionar sobre su aplicabilidad para la comprensión de fenómenos psicosociales que ocurren en el ambiente escolar. Suponemos que el lenguaje verbal tiende a favorecer las normas sociales más influyentes en el ambiente académico y fuera del mismo, perjudicando la expresión de individuos y grupos que adoptan otras normas, supuestamente menos legítimas y/o no reconocidas socialmente. Los participantes fueron estudiantes de enseñanza primaria y media de escuelas públicas de la ciudad de Rio de Janeiro. Ellos hicieron un dibujo de la escuela donde estudiaban y, seguidamente, otro dibujo sobre el interior de la misma $y_{\text {, }}$ finalmente, se expresaron sobre los dibujos hechos. Constatamos tendencias de valorización positiva de la imagen de la escuela y menor envolvimiento con actividades típicas de enseño y aprendizaje. La escuela dibujada indicó la necesidad de un trabajo de dinámica grupal en el ambiente escolar para poder insertar a los estudiantes. Palabras clave: Evaluación escolar. Dibujo de la escuela. Psicología social de la educación. 


\section{Introdução}

A atividade de avaliação em Educação, sendo ela específica ou mais geral, tornou-se cada vez mais necessária para a superação de problemas nesse campo. Tendo em vista a situação de crescimento da aspiração de democratização das relações sociais no ambiente escolar, a importância da consulta de todos os envolvidos no processo educacional tornou-se um dos maiores desafios. Em geral, tem havido mais ênfase nos métodos "racionais" de avaliação, tais como o questionário autoadministrado ou outras formas de expressão verbal, como a associação livre. Tais métodos têm obtido resultados muito valiosos para o aperfeiçoamento da área de Educação, porém eles costumam veicular conteúdos comportamentais e mentais muito marcados por normas sociais. Numa sociedade em que existem vários grupos sociais que regularmente não conseguem inserir-se de modo adequado no ambiente escolar, o desenho permitiria acessar, justamente, alguns conteúdos, muitos dos quais latentes ou impedidos de expressão, devido a dificuldades de negociação entre indivíduos e grupos mais ou menos influentes. Nesse sentido, uma pesquisadora italiana já havia constatado um grande contraste, ao estudar atitudes em relação ao doente mental na Itália, por meio de questionários autoadministrados e desenhos, sobretudo entre os de mais escolaridade e idade, que apresentavam mais atitudes favoráveis por meio de questionário e mais desfavoráveis através de desenhos (DE ROSA, 1987). Segundo a autora, tais fatos se deviam à tendência de construção racional de autoimagem de acordo com o que o establishment acadêmico preconizava publicamente, ou seja, de inclusão social do doente mental, ao lado da influência da cultura iconográfica italiana tradicional, impregnada de conteúdos desfavoráveis a respeito da doença mental. Acreditamos que os conteúdos subjetivos mencionados existentes no repertório de envolvidos no ambiente escolar tenham um impacto importante no que ocorre ali, cabendo incorporá-los a partir de outros instrumentos de avaliação em Educação, tais como o desenho. 0 objetivo geral deste trabalho foi realizar uma investigação comparativa a respeito da avaliação da escola pública feita por estudantes de fundamental e médio por meio de desenho na cidade do Rio de Janeiro.

A educação tem passado por grandes transformações, resultado de empreendimentos diversos em vários campos da vida social e política, no Brasil e em outros países. Parte do que tem ocorrido em termos de mudanças de concepções e práticas, decorre de buscas sociais, pedagógicas, psicossociais, entre outras, as quais têm-se refletido, inclusive, no modo de construção arquitetônica das escolas. Aliado a isso, indivíduos/grupos comuns procuram realizar aspirações e projetos, sociais e educacionais, ao se dirigirem aos diferentes estabelecimentos de ensino, onde vivem experiências psicossociais importantes, a serem mais conhecidas a partir de pesquisa. Parte do que muitos envolvidos vivem no ambiente escolar foi elaborado por outros personagens, tais como formuladores de políticas públicas, acadêmicos, políticos, dirigentes e funcionários educacionais. Tais empreendimentos educacionais são reconstruí- 
dos pelos não especialistas na forma de representações sociais, que são conhecimentos práticos elaborados para pensar e agir na vida cotidiana. Assim, nosso objetivo específico nesse trabalho foi mapear representações sociais da escola através de desenhos e textos de estudantes do ensino médio e fundamental, segundo o tipo de instituição e nível de ensino na zona metropolitana da cidade do Rio de Janeiro.

Adotamos como referencial teórico o proposto por Moscovici (1976), segundo o qual indivíduos/grupos comuns na situação urbana e moderna constantemente se veem diante de objetos estranhos e não familiares como a escola, sobre a qual produzem representações sociais a fim de a conhecerem e transformarem, segundo seus valores, normas e finalidades sociais e psicossociais. De acordo com o mesmo autor, os processos de construção social de representações sociais apresentam dois aspectos fundamentais: materialização do objeto de representação e sua inserção no universo de significados e práticas, oriundas do ambiente social e psicológico de indivíduos e grupos.

0 estudo de representações sociais em Educação em outros países tem obtido resultados promissores (GILLY, 1984; EMLER, OHANA; MOSCOVICI, 1987), e também no Brasil (ALVES-MAZZOTTI, 1994; COSTA; ALMEIDA, 1998). Contudo, poucos trabalhos a partir da teoria das representações sociais foram feitos utilizando o desenho como forma de expressão (GALLI; NIGRO, 1987; DE ROSA, 1987), cabendo mais investigações. Ao lado disso, alguns autores têm adotado o desenho no campo da Educação, ainda que a partir de outros referenciais teóricos (WEBER; MITCHELL, 1996). Enfim, podemos dizer que os métodos projetivos em Psicologia têm dado mais importância aos conteúdos latentes, não acessiveis através de instrumentos que reproduzem situações e contextos em que existe presença de normas sociais convencionais (ANZIEU, 1979). Nesse sentido, o próprio espaço formulado e construído por protagonistas do ambiente escolar e vivido por professores e alunos pode-se tornar um objeto de representações sociais (SALES, 2000; 2002; ESOUIVEL, 1997; LOURENÇO, 2005).

É preciso dizer que o espaço vivido tem sido investigado em ciências humanas a partir de outros referenciais teóricos (SOMMER, 1969; MILGRAM, 1984), assim como especificamente o ambiente escolar (GUMP, 1987; VAYER, DUVAL; RONCIN, 1993; FISCHER, 1980, 1989). Em termos psicológicos e sociais, podemos supor que, mesmo que o espaço físico já contenha a subjetividade de quem os construiu, ele é transformado em termos psicossociais pelos que estão nele inseridos.

Apesar de estar presente em quase todo o pais e fazer (ou ter feito) parte do cotidiano de quase todas as famílias, podemos dizer que a escola é um ambiente social problemático para muitos brasileiros, tendo em vista as dificuldades enfrentadas por muitos individuos/grupos sociais na sociedade para realizar os objetivos educacionais propostos e, muitas vezes, impostos por profissionais e acadêmicos. Existe uma aspi- 
ração à educação entre muitos brasileiros, mas sua realização tem sido inferior aos recursos humanos e materiais disponibilizados. Podemos dizer que uma das principais dificuldades educacionais no Brasil é a pouca escolarização dos brasileiros em geral, criando uma espiral de atraso que pode manter-se indefinidamente se não conseguirmos conhecer e modificar o fenômeno adequadamente. Trata-se de um fenômeno produto de múltiplas causas. Contudo, consideramos que há menos estudos sobre a experiência psicossocial vivida no ambiente sociofísico da escola. Tendo em vista o acúmulo de experiências no ambiente escolar mais de frustração que de realização entre muitos brasileiros, seria interessante trabalharmos as representações sociais da escola através de desenhos, o que nos permitiria acessar os conteúdos subjetivos menos influenciados pelos discursos/práticas dominantes relativos à educação formal.

\section{Método}

Os participantes foram estudantes de estabelecimento públicos do Estado do Rio de Janeiro, de ensino fundamental e médio, de ambos os sexos. A pesquisa foi financiada pelo CNPq, em projeto bianual entre 1998-1999, após o que os dados foram reanalisados e apresentados em formato abreviado num congresso nacional em 2008.

Nosso objetivo principal foi fazer um estudo comparativo de representações e práticas sociais em diferentes ambientes escolares a partir de uma base comum de análise, relacionada aos conteúdos que emergissem espontaneamente do material produzido por alunos para desenhar a escola. Partimos da hipótese de que não existiriam diferenças significativas entre os conjuntos de desenhos feitos nos diferentes estabelecimentos em termos de menor ênfase em ensino-aprendizagem e maior em aspectos físico-espaciais das edificações e outros fenômenos de vivência individual/ social em espaços públicos, independente das políticas pedagógicas preconizadas nos estabelecimentos comparados. Para tanto, procuramos selecionar escolas públicas relevantes e contrastantes cronologicamente, no sentido de funcionarem em prédios construídos em momentos distintos e em função de políticas públicas diferenciadas na história da educação da cidade. Assim, trabalhamos com estudantes do ensino médio do Colégio Pedro II (CPII) ( $n=19)$ (média de idade de 15 anos), do Instituto de Educação de São João do Meriti (IESJM) (n=69) (média de idade de 16 anos) e do ensino fundamental do Estado do Rio de Janeiro (EFRJ) $(n=36)$ (média de idade de 16,3 anos). Solicitamos que os estudantes fizessem um desenho da escola onde estudavam, incluindo tudo o que ele/ela quisessem incluir. Para tanto, folhas de papel em branco foram-Ihes oferecidas. Em seguida, propusemos que fizessem um desenho do interior da escola, segundo o mesmo procedimento anterior. A pesquisa foi realizada em sala de aula, coletivamente, mediante acordo com os estabelecimentos e os alunos que aceitassem, voluntariamente, participar. 0 material desenhado e verbalizado foi analisado em termos temáticos (BARTHES, 1957; BARDIN, 1991; DE MĖREDIEU, 1990; JOLY, 1993; VIGOSKII, 1996) e estatísticos através de testes de qui-quadrados para comparar as escolas mencionadas. 
Foram encontrados os seguintes temas gerais, os quais se basearam, sobretudo, nos desenhos como veículo de expressão/comunicação: locais do estabelecimento: entrada/fachada, circulação, sala de aula, biblioteca, lazer, alimentação, banheiro, administração, serviços, espaço do aluno; papéis escolares/indivíduos/relações: alunos/colegas, amigos, professor, administração/funcionário, indivíduo; objetos escolares: porta/janela, vaso/planta/árvore, conforto ambiental, símbolo institucional, equipamento escolar, equipamento do edifício, instrumento do professor, instrumento do aluno, comunicação/transporte, mural; perspectivas espaciais: de cima para baixo, em frente, estrutura, andar/parte, diagonal/lateral, acabamento, natureza/rua; inscrições verbais: apropriação individual/coletiva, greve, pichação, falta de manutenção, matar aula.

Apresentamos em seguida alguns fac-símiles dos desenhos analisados.

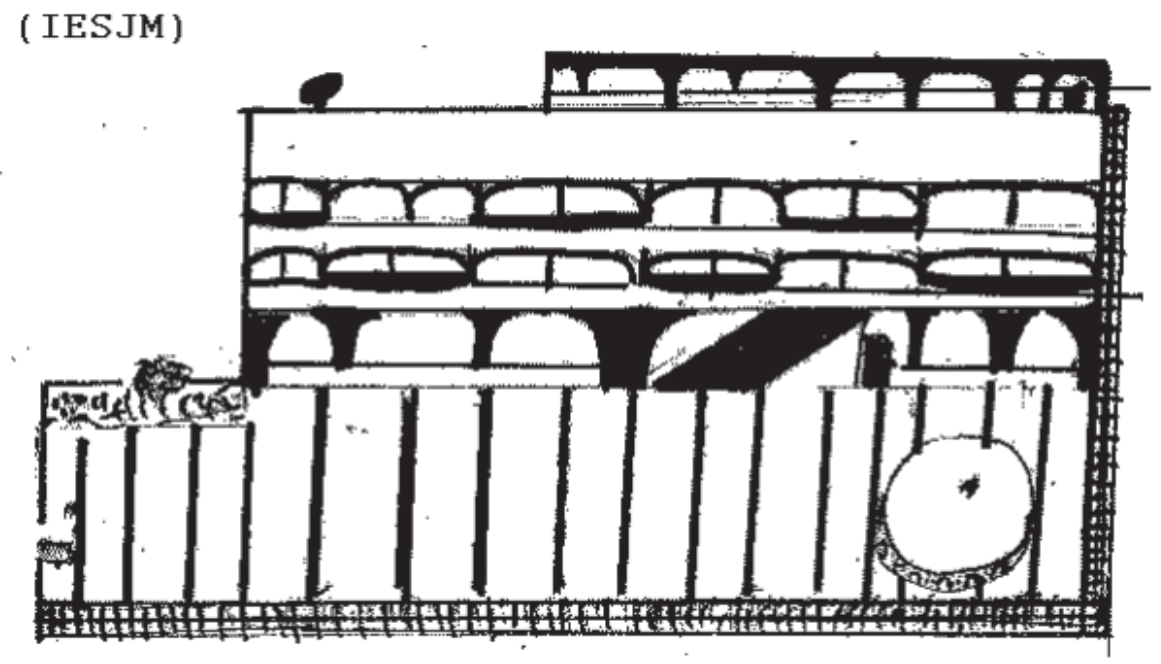

Figura 1: Desenho de aluno do Instituto de Educação de São João d̄o Meriti (IESJM). 


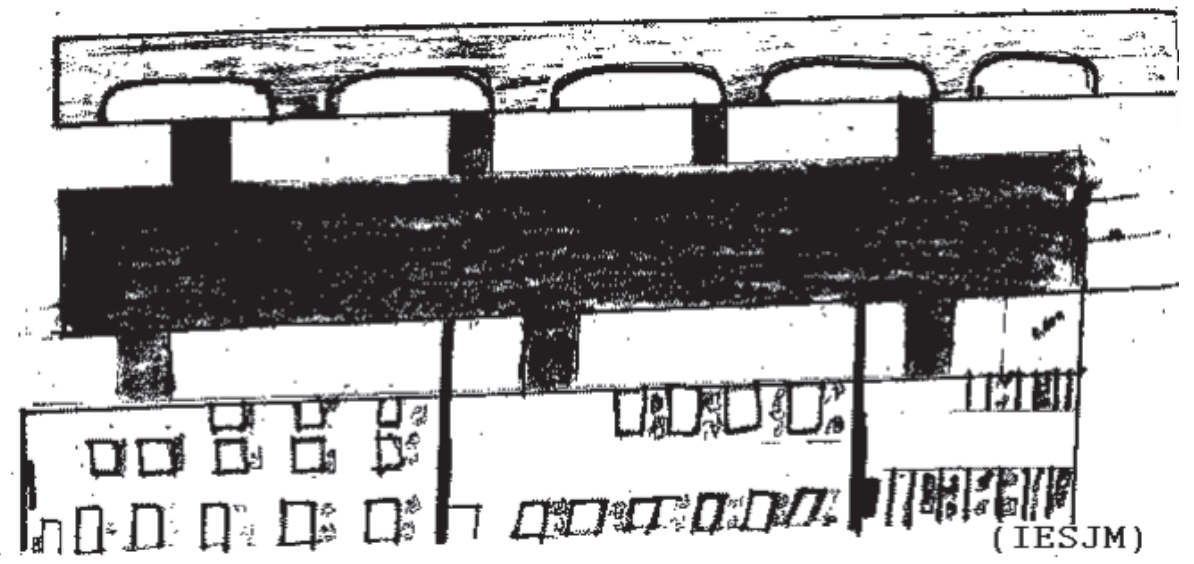

Figura 2: Desenho de aluno do Instituto de Educação de São João do Meriti (IESJM).

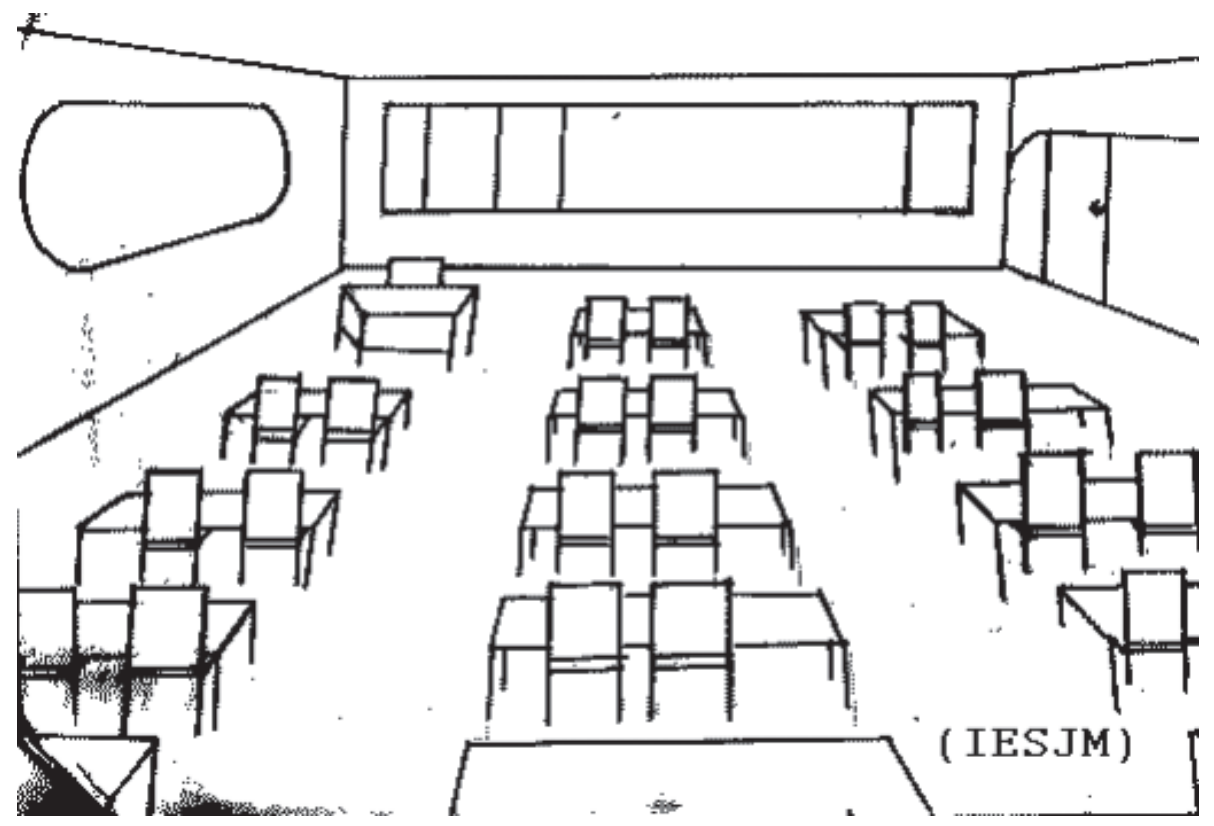

Figura 3: Desenho de aluno do Instituto de Educação de São João do Meriti (IESJM). 

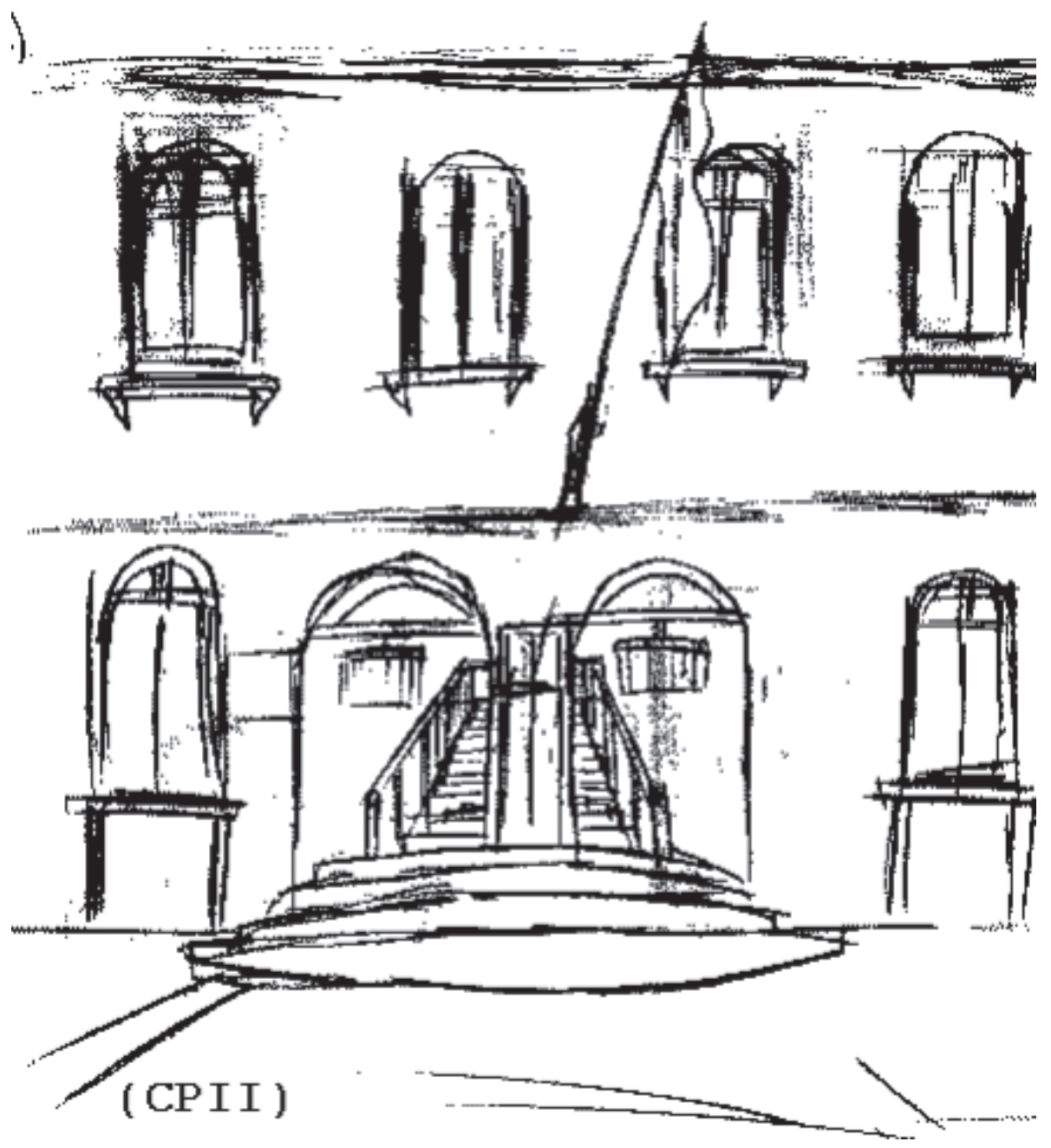

Figura 4: Desenho de aluno do Colégio Pedro II (CPII). 


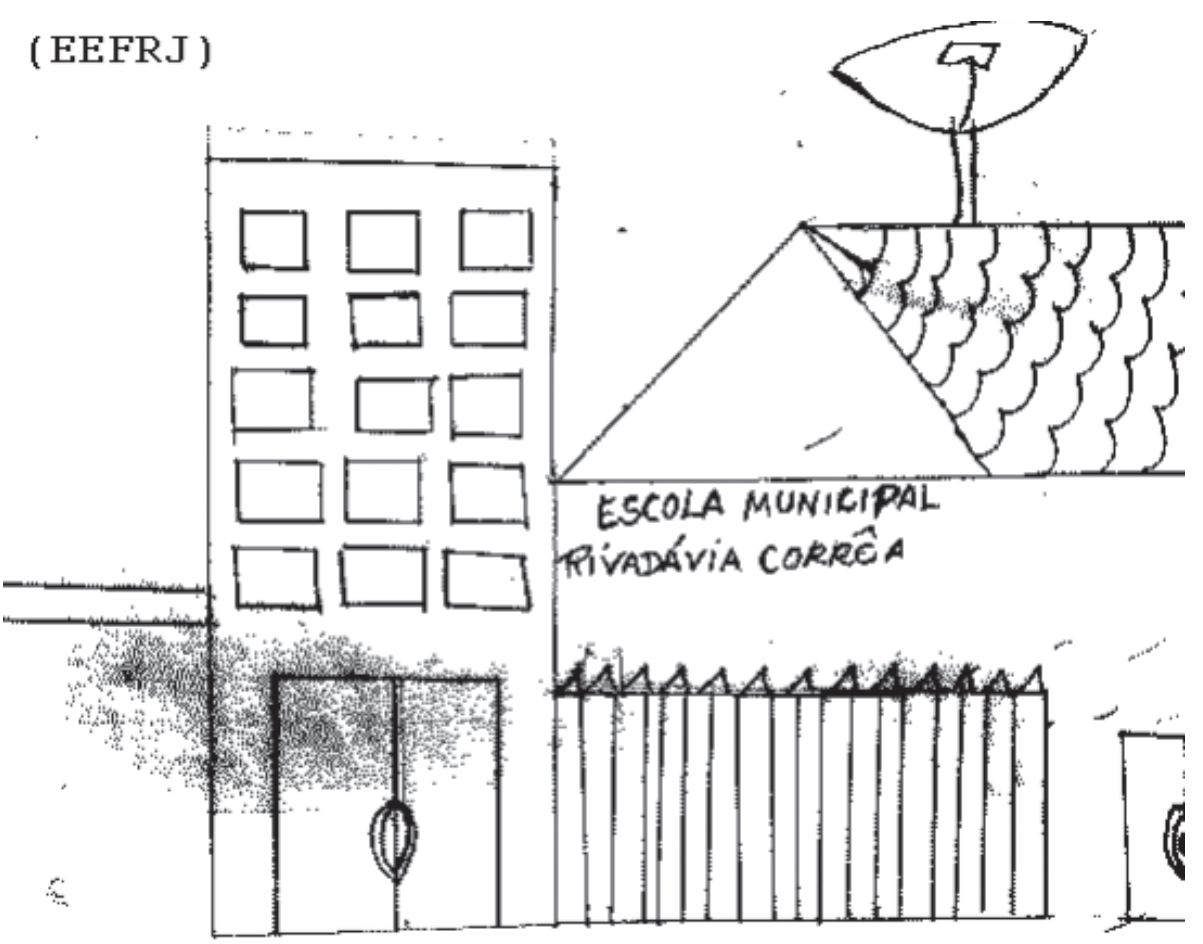

Figura 5: Desenho de aluno do Ensino Fundamental do Estado do Rio de Janeiro (EEFRJ).

\section{Resultados}

Abaixo apresentamos tabelas com os principais resultados, incluindo conteúdos referentes aos locais do estabelecimento (Tabela 1), aos objetos escolares (Tabela 2), às perspectivas espaciais (Tabela 3), assim como os referentes ao interior da escola a respeito dos locais do estabelecimento (Tabelas 4), dos objetos escolares (Tabela 5), das perspectivas espaciais (Tabelas 6) e dos papéis escolares/individuos/relações (Tabelas 7). 
Tabela 1: Frequências e percentagens de temas usados para representar os locais da escola pública no Rio de Janeiro, segundo o tipo de escola.

\begin{tabular}{|c|c|c|c|c|c|c|}
\hline \multirow[t]{2}{*}{ Temas/escolas } & \multicolumn{2}{|c|}{ CPII } & \multicolumn{2}{|c|}{ EEFRJ } & \multicolumn{2}{|c|}{ IESJM } \\
\hline & $\mathrm{F}$ & $\%$ & $\mathrm{~F}$ & $\%$ & $\mathrm{~F}$ & $\%$ \\
\hline Sala de aula & 0 & 0,0 & 6 & 8,45 & 1 & 0,51 \\
\hline Banheiro & 0 & 0,0 & 3 & 4,22 & 5 & 2,57 \\
\hline Biblioteca & 0 & 0,0 & 1 & 1,40 & 23 & 11,86 \\
\hline Refeitório/cantina & 0 & 0,0 & 1 & 1,40 & 14 & 7,21 \\
\hline Pátio/piscina/quadra & 5 & 9,09 & 13 & 18,31 & 50 & 25,77 \\
\hline Telhado/laje & 21 & 38,18 & 32 & 45,07 & 43 & 22,16 \\
\hline Administ./sala professor & 0 & 0,0 & 1 & 1,40 & 1 & 0,51 \\
\hline Circulação & 29 & 52,72 & 11 & 15,49 & 52 & 26,80 \\
\hline Espaço do aluno & 0 & 0,0 & 1 & 1,40 & 2 & 1,03 \\
\hline Serviço & 0 & 0,0 & 2 & 2,81 & 3 & 1,54 \\
\hline
\end{tabular}

Nota: $X=73,543 ; g l=18 ; p<0,0000$

A Tabela 1 acima trata da escola em termos de locais (incluindo a própria edificação e seu entorno imediato). Pela análise que fizemos, o registro do aspecto físico-espacial (telhado/laje) foi priorizado no CPII e EEFRJ, mas os três tipos de edificação geraram grande concentração de respostas relacionadas à circulação, serviços de lazer/esporte (pátio/piscina/quadra) e satisfação de necessidades básicas (refeitório/cantina e banheiro) não diretamente ligadas ao ensino/aprendizagem.

Tabela 2: Frequências e percentagens de temas usados para representar objetos da escola pública no Rio de Janeiro, segundo o tipo de escola.

\begin{tabular}{lrrrrrr}
\hline Temas/escolas & \multicolumn{1}{c}{ CPII } & \multicolumn{2}{c}{ EEFRJ } & \multicolumn{3}{c}{ IESJM } \\
& $\mathrm{F}$ & $\%$ & $\mathrm{~F}$ & $\%$ & $\mathrm{~F}$ & $\%$ \\
\hline Instrumento professor & 1 & 1,14 & 1 & 1,33 & 0 & 0,0 \\
\hline Porta/janela & 49 & 56,32 & 58 & 77,33 & 162 & 49,39 \\
\hline Conforto & 0 & 0,0 & 4 & 5,33 & 117 & 35,67 \\
\hline telefone/transporte & 2 & 2,29 & 3 & 4,00 & 27 & 8,23 \\
\hline Vaso/planta/flor & 10 & 11,49 & 2 & 2,67 & 8 & 2,43 \\
\hline Símbolo institucional & 7 & 8,04 & 0 & 0,0 & 0 & 0,0 \\
\hline Equipamen. educacional & 5 & 5,74 & 0 & 0,0 & 6 & 1,82 \\
\hline Equipamen. Físico & 7 & 8,04 & 1 & 1,33 & 3 & 0,91 \\
\hline Outros & 6 & 6,90 & 6 & 8,00 & 5 & 1,52 \\
\hline
\end{tabular}

Nota: $X=146,478 ; g l=16 ; p<0,0000$

A tabela 2, acima, reúne aspectos mais relacionados à atividade ensino/aprendizagem (instrumento do professor e equipamento educacional), mas houve mais desta- 
que seja para aspectos físico-espaciais (porta/janela) e conforto ambiental, seja para os aspectos simbólicos e decorativos (símbolo institucional e vaso/planta/flor).

Tabela 3: Frequências e percentagens de temas usados para representar perspectivas espaciais da escola pública no Rio de Janeiro, segundo o tipo de escola.

\begin{tabular}{lrrrrrr}
\hline Temas/escolas & \multicolumn{2}{c}{ CPII } & \multicolumn{2}{c}{ EEFRJ } & \multicolumn{3}{c}{ IESJM } \\
& $\mathrm{F}$ & $\%$ & $\mathrm{~F}$ & $\%$ & $\mathrm{~F}$ & $\%$ \\
\hline Cima para baixo & 3 & 4,84 & 8 & 16,67 & 6 & 3,87 \\
\hline Em frente/fachada & 18 & 29,03 & 14 & 29,17 & 52 & 33,55 \\
\hline Estrutura/pilastra & 5 & 8,06 & 1 & 2,08 & 34 & 21,93 \\
\hline Andar/parte & 7 & 11,29 & 14 & 29,17 & 42 & 27,10 \\
\hline Diagonal/lateral & 1 & 1,61 & 3 & 6,25 & 2 & 1,29 \\
\hline Acabamento & 10 & 16,13 & 1 & 2,08 & 7 & 4,51 \\
\hline Natureza & 8 & 12,90 & 7 & 14,58 & 8 & 5,16 \\
\hline Calçada & 10 & 16,13 & 0 & 0,0 & 4 & 2,58 \\
\hline
\end{tabular}

Nota: $X=66,398 ; g l=14 ; p<0,0000$

A tabela 3 acima indica novamente o físico-espacial como o elemento predominante para tratar de fachada ou detalhes, referidos em função do próprio prédio em termos objetivos (como estrutura/pilastra ou andar/parte). Contudo, tiveram destaque também acabamentos, complementos paisagísticos (natureza e calçada).

Tabela 4: Frequências e percentagens de temas usados para representar locais no interior da escola pública no Rio de Janeiro, segundo o tipo de escola.

\begin{tabular}{|c|c|c|c|c|c|c|c|c|c|}
\hline \multicolumn{3}{|c|}{ Temas/escolas } & \multicolumn{3}{|l|}{ CPII } & \multicolumn{2}{|l|}{ EEFRJ } & \multirow{2}{*}{$\begin{array}{l}\text { IESJM } \\
\mathrm{F} \\
\end{array}$} & \multirow[b]{2}{*}{$\%$} \\
\hline & & & $\mathrm{F}$ & \multicolumn{2}{|c|}{$\%$} & & $\%$ & & \\
\hline Sala de aula & 6 & 15,79 & 31 & 31,63 & 61 & 38,85 & & & \\
\hline Banheiro & 0 & 0,0 & 7 & 7,14 & 25 & 15,92 & & & \\
\hline Biblioteca & 0 & 0,0 & 1 & 1,02 & 7 & 4,46 & & & \\
\hline Refeitório/ca & ntina & 2 & 5,26 & 7 & 7,14 & 2 & 1,27 & & \\
\hline Pátio/piscina & /quadra & 17 & 44,74 & 11 & 11,22 & 5 & 3,18 & & \\
\hline Telhado/laje & 3 & 7,90 & 9 & 9,18 & 4 & 2,55 & & & \\
\hline Entrada/port & & 0 & 0,0 & 4 & 4,08 & 7 & 4,45 & & \\
\hline Administr./sa & la profes. & & 0,0 & 5 & 5,10 & 3 & 1,91 & & \\
\hline Circulação & 8 & 21,05 & 11 & 11,22 & 36 & 22,93 & & & \\
\hline Espaço do alı & uno & 1 & 2,63 & 0 & 0,0 & 1 & 0,63 & & \\
\hline Serviço & 1 & 2,63 & 2 & 2,04 & 5 & 3,18 & & & \\
\hline Outros & 0 & 0,0 & 10 & 10,20 & 1 & 0,63 & & & \\
\hline
\end{tabular}

Nota: $X=104,944 ; g l=22 ; p<0,0000$ 
A tabela 4 expõe os locais do interior dos prédios escolares. Novamente foram confirmadas as tendências encontradas no desenho da escola ( $1^{\circ}$. Desenho) feito pelo participante, de aspectos não relacionados ao ensino/aprendizagem.

Tabela 5: Frequências e percentagens de temas usados para representar objetos no interior da escola pública no Rio de Janeiro, segundo o tipo de escola.

\begin{tabular}{lrrrrrr}
\hline Temas/escolas & \multicolumn{2}{l}{ CPII } & \multicolumn{2}{c}{ EEFRJ } & \multicolumn{3}{c}{ IESJM } \\
& $\mathrm{F}$ & $\%$ & $\mathrm{~F}$ & $\%$ & $\mathrm{~F}$ & $\%$ \\
\hline Instrumento professor & 7 & 7,52 & 9 & 12,86 & 50 & 17,54 \\
\hline Instrumento aluno & 1 & 1,07 & 14 & 20,00 & 30 & 10,52 \\
\hline Porta/janela & 23 & 24,73 & 9 & 12,86 & 108 & 37,90 \\
\hline Conforto ambiental & 5 & 5,37 & 6 & 8,57 & 15 & 5,26 \\
\hline Telefone/carro & 0 & 0,0 & 1 & 1,43 & 0 & 0,0 \\
\hline Mural & 2 & 2,15 & 1 & 1,43 & 4 & 1,40 \\
\hline Vaso/planta/flor/arvore & 18 & 19,35 & 0 & 0,0 & 3 & 1,05 \\
\hline Simbolo institucional & 6 & 6,45 & 0 & 0,0 & 0 & 0,0 \\
\hline Equipamen. educacional & 23 & 24,73 & 22 & 31,43 & 44 & 15,44 \\
\hline Equipamen. Físico & 5 & 5,37 & 4 & 5,71 & 26 & 9,12 \\
\hline Objeto refeitório/cantina & 3 & 3,23 & 4 & 5,71 & 5 & 1,75 \\
\hline
\end{tabular}

Nota: $X=129,7 ; g l=20 ; p<0,0000$

A tabela 5 acima apresenta dados sobre objetos, em que se destacaram aspectos não diretamente relacionados ao ensino/aprendizagem - com exceção da EERJ cuja frequência de temas instrumento do professor e aluno, equipamento educacional foi relativamente mais alta que nas demais escolas - ou seja, foram mais frequentes os aspectos relacionados à circulação (porta/janela), decoração (vaso/planta/árvore) e equipamento físico ou serviços para atender necessidade básica (objeto refeitório/cantina).

Tabela 6: Frequências e percentagens de temas usados para representar pessoas/papéis sociais no interior da escola pública no Rio de Janeiro, segundo o tipo de escola.

\begin{tabular}{llrrrrr}
\hline Temas/escolas & \multicolumn{2}{l}{ CPII } & \multicolumn{2}{c}{ EEFRJ } & \multicolumn{3}{c}{ IESJM } \\
& $\mathrm{F}$ & $\%$ & $\mathrm{~F}$ & $\%$ & $\mathrm{~F}$ & $\%$ \\
\hline Professor & 0 & 0,0 & 3 & 30,0 & 2 & 6,25 \\
\hline Colega/amigo & 5 & 33,33 & 0 & 0,0 & 9 & 28,12 \\
\hline Aluno & 7 & 46,67 & 6 & 60,0 & 14 & 43,75 \\
\hline Coordenador & 0 & 0,0 & 0 & 0,0 & 4 & 12,50 \\
\hline Administr./funcionário & 0 & 0,0 & 1 & 10,0 & 3 & 9,37 \\
\hline Outros & 3 & 20,0 & 0 & 0,0 & 0 & 0,0 \\
\hline
\end{tabular}

Nota: $\mathrm{X}=$ não significativo 
A tabela 6 reúne pessoas/papéis sociais que foram incluídos no desenho. Em geral, as frequências foram proporcionalmente menores em relação a outros temas analisados para os estabelecimentos comparados, indicando a existência de paisagens vazias de sujeitos. Houve maior destaque para os próprios alunos e, em segui$\mathrm{da}$, colegas/amigos, em contraste com o professor que foi relativamente menos frequente, com exceção da EERJ em que houve o inverso, ou seja, menor presença do colega/amigo e maior do professor.

Tabela 7: Frequências e percentagens de temas usados para representar perspectivas do interior da escola pública no Rio de Janeiro, segundo o tipo de escola.

\begin{tabular}{|c|c|c|c|c|c|c|}
\hline \multirow[t]{2}{*}{ Temas/escolas } & \multicolumn{2}{|c|}{ CPII } & \multicolumn{2}{|c|}{ EEFRJ } & \multicolumn{2}{|c|}{ IESJM } \\
\hline & $\mathrm{F}$ & $\%$ & $\mathrm{~F}$ & $\%$ & $\mathrm{~F}$ & $\%$ \\
\hline Cima para baixo & 11 & 27,50 & 14 & 60,87 & 36 & 39,56 \\
\hline Em frente/fachada & 3 & 7,50 & 7 & 30,43 & 23 & 25,27 \\
\hline Estrutura/pilastra & 2 & 5,00 & 0 & 0,0 & 16 & 17,58 \\
\hline Andar/parte & 6 & 15,00 & 1 & 4,35 & 9 & 9,89 \\
\hline Diagonal/lateral & 4 & 10,00 & 1 & 4,35 & 1 & 1,10 \\
\hline Acabamento & 8 & 20,00 & 0 & 0,0 & 6 & 6,59 \\
\hline Natureza & 6 & 15,00 & 0 & 0,0 & 0 & 0,0 \\
\hline
\end{tabular}

Nota: $X=48,514 ; g l=12 ; p<0,0000$

A tabela 7, enfim, reúne os temas a respeito das perspectivas físico-espaciais do interior das escolas. Houve grande concentração de desenhos retratando perspectivas afastadas, vale dizer, de um espectador que toma distância em relação ao prédio como de cima para baixo, em frente/fachada, assim como perspectivas mais ligadas ao prédio em termos de estrutura (estrutura/pilastra e andar/parte) e aspectos de acabamento.

Como acabamos de relatar, em geral, o modo de representar socialmente a escola seja do objeto "escola como um todo", em termos arquiteturais e ambientais, seja do uso do prédio, em termos individuais e sociais, em termos de perspectivas espaciais, observamos diferenciação entre as escolas e aproximações. Houve aproximações quanto à perspectiva focalizando a frente da escola, com algumas exceções. Podemos supor que os estudantes tentaram fazer um "retrato" da frente das escolas, sendo que os do Colégio Pedro II e algumas escolas públicas preferiram focalizar aspectos que consideravam importantes em cada estabelecimento, tal como os detalhes da fachada que indicassem aspectos históricos considerados como símbolos de distinção institucional do primeiro e a natureza em termos de árvores e plantas do segundo. Já os alunos do Instituto de Educação de São João de Meriti, por sua vez, tenderam a ressaltar a estrutura peculiar da sua arquitetura de tipo CIEP, reforçada pelos detalhes dos andares da escola, assim como em outras procuraram mostrar o prédio a partir de um olhar de sobrevoo, de cima para baixo, como uma linguagem de reportagem de TV. 
Em termos de uso, os locais da escola referidos indicaram duas grandes tendências gerais: uma relacionada ao ensino-aprendizagem (sala de aula) e outra de circulação do estudante no ambiente escolar (corredores/rampas/escadas). Contudo, foi muito mencionado outro conjunto de dados relacionados ao lazer do estudante (pátios/ terraços/piscinas), ao que deveriamos acrescentar os relativos à alimentação e higiene. Enfim, um último conjunto é o que diz respeito aos papéis escolares/indivíduos/relações, bem como aos objetos que eles utilizam dentro da escola. Em geral, os estudantes se referiram mais a objetos do que a papéis escolares/indivíduos/relações. Contudo, houve mais destaque para equipamentos escolares, portas/janelas e instrumentos dos alunos e professores. Entre os papéis, houve destaque para os alunos/indivíduos, professores e amigos/colegas

\section{Discussão}

Consideramos que os objetivos da pesquisa foram atingidos, uma vez que o desenho permitiu-nos acessar alguns aspectos globais e específicos relativos aos diferentes modos de elaboração/inserção psicossocial adotados por estudantes de escolas públicas da zona metropolitana da cidade do Rio de Janeiro. A pesquisa indicou-nos dois aspectos importantes da construção social da escola entre estudantes: de busca de uma imagem pública positiva da escola na sociedade e, outra, de menor participação em atividades típicas de ensino-aprendizagem.

Em primeiro lugar, cabe lembrar que os estudantes receberam instruções bastante abertas, sendo que foi mencionado o fato de que ele/ela estudava na escola sobre a qual faria um desenho. Tratou-se, portanto, de uma construção livre de uma experiência com um ambiente construído, para a qual a dimensão psicossocial desempenhou um papel relevante. Nesse sentido, os estudantes das escolas observadas apresentaram uma diferenciação considerável entre si diante da situação proposta de fazer um desenho da escola. Poderíamos dizer que tanto os do Colégio Pedro II e quanto do Instituto de Educação de São João de Meriti - o qual vale lembrar funciona em um prédio do tipo CIEP -, dispõem de distinções públicas relativamente favoráveis, que podem ser aspiradas por estudantes como formas de distinção individual (BOURDIEU, 1979; 1987). 0 primeiro por ter mantido um prestígio prolongado de qualidade educacional e o segundo, por ter tido como origem um projeto educacional de busca de qualidade, que foi representado pela arquitetura especifica adotada. Acreditamos que, diante de uma incerteza sobre a realização dos objetivos educacionais e sociais relacionados, os estudantes das escolas mencionadas preferiram ressaltar esses aspectos imaginários que supõem existir entre muitos na sociedade, indicando expectativas sociais positivas. Contudo, os estudantes do Colégio Pedro II e foram justamente os que menos mencionaram tanto a sala de aula, quanto às atividades/objetos relacionados ao ensino-aprendizagem, indicando certa dispersão e/ou acomodação, merecendo reflexões. Ou seja, poderíamos nos perguntar se os objetivos educacionais de muitos estudantes não estariam cen- 
trados na construção de uma autoimagem individual positiva, em que o diploma de uma instituição reconhecida poderia ser uma garantia.

Parte dos resultados descritos indicou que existem regularidades importantes entre os estudantes observados. Apesar de os estudantes estarem em faixa etária próxima, eles apresentaram modos diferenciados de representar a escola antes de tudo em função da inserção, seja no ensino fundamental, seja no médio. Ora, sabemos que durante a entrada no ensino médio existe certa tendência de autonomia, de dispersão de atenção, propiciando toda uma busca de outras fontes de estimulação, que pode ter gerado uma parte do que observamos. Já os estudantes no final do ensino fundamental, devido a certo ambiente de maior controle social externo, quando ainda não existe certeza de uma carreira acadêmica bem sucedida, pode haver uma expectativa de subordinação e focalização na relação aluno e professor. Outros estudos que pretendemos realizar futuramente poderão precisar mais a natureza dos fenômenos observados. Tratar-se-ia de saber, portanto, se a crença de sucesso acadêmico entre estudantes brasileiros poderia afetar os resultados que obtivemos, tanto no sentido de se permitir uma dispersão quanto de maior busca de concentração nas atividades escolares. Contudo, os resultados obtidos em um estudo correlacional feito na Romênia a partir do mesmo procedimento que adotamos no Rio de Janeiro, indicaram que, naquele país, os estudantes com desempenho acadêmico mais alto tenderam a focalizar mais aspectos de ensino-aprendizagem nos seus desenhos da escola (ESQUIVEL, 1997).

Por outro lado, alguns estudos de psicologia social do espaço indicaram a saliência psicológica dos aspectos físicos do meio ambiente para alguns grupos sociais (FISCHER, 1989), particularmente em relação ao corpo (JODELET, 1994), em detrimento de outras elaborações relacionadas ao indivíduo ou grupo social. Ou seja, alguns grupos tenderiam a focalizar mais o mundo externo em detrimento do interno.

Acreditamos que esses fenômenos poderiam ser atenuados a partir de uma reeducação de vivência interativa entre corpo e meio ambiente físico, o que poderia ser tratado por meio de materiais didáticos, visuais ou outros. Nesse sentido, a presença de janelas e portas em proporções altas poderia indicar uma tendência de evasão psicológica do local ou de dispersão mental em relação aos objetivos centrais do estabelecimento. Tratar-se-ia, por exemplo, de deslocar a atenção de fora para dentro a partir de exercícios de concentração, inicialmente voltados para uma relação consigo mesmo, fechando os olhos e procurando afastar-se do ambiente físico e social imediato para, em seguida, retomar para as diferentes tarefas enfrentadas, entre outras possibilidades.

É possível que as concepções da atividade educacional estejam em momento de mutação, havendo a presença de competição mercadológica com outras atividades, inclusive as de compras e lazer, as quais são atividades livres e sem compromisso de 
disciplina. Nesse sentido, nos desenhos analisados foram muito mencionados, simultaneamente, a circulação (como se faz nos corredores de Shoppings) e o lazer social e contemplativo (como nos clubes e lugares de diversão). Trata-se de um desafio para os educadores lidar com essa situação. Aparentemente, eles teriam que competir em termos publicitários com outras atividades individuais e coletivas (LE BON, 1985; GRAUMANN; KRUSE, 1984), cabendo mais pesquisas sobre o assunto, inclusive a respeito de outros recursos de tecnologia de informação, que trabalham de modo mais explícito a distração/imaginação, entre outras dimensões. Nesse sentido, é possível que a atividade de desenhar esteja associada a lazer, distração, entre outras, consideradas menos acadêmicas, porém equivalentes a formas de apropriação individual e social (GRAUMANN, 1979), explicando parte do que obtivemos. Mas os resultados acima relatados poderiam causar alguma preocupação, uma vez que as atividades de ensino-aprendizagem ficaram em muito menor proporção do que se poderia esperar nos conteúdos dos desenhos analisados.

Nesse sentido, estudos de comportamento de jovens feitos em ambientes escolares e públicos no Brasil, e em outros países indicaram a tendência de opressão (PATTO, 1980) e anomia social entre os mesmos, tendendo a atingir seriamente as atividades em Educação na forma de vandalismo, agressão à autoridade, aos colegas, às minorias sociais presentes na escola, entre muitos outros. Tais atividades não foram explicitamente captadas pela pesquisa aqui relatada, mas o comportamento dos estudantes aparentemente anódino de estarem mais envolvidos com atividades não diretamente escolares indica o início do fenômeno mencionado, apesar de eventualmente obedecerem a outras normas sociais (MARSH, ROSSER; HARRÉ, 1978). Trata-se de uma dinâmica grupal do tipo minorias versus maiorias (LEWIN, 1948; TAJFEL, 1981), no sentido de indivíduos e grupos menos/mais valorizados na sociedade, dotados de menos/mais legitimidade, o que implica sentimentos de incapacidade/capacidade para interferir no ambiente escolar de alguma maneira (TAJFEL, 1981). Acreditamos que cada escola pode dinamizar sua vida interna (TEDESCO, 1996), o que pode ser precedido por consultas do tipo que fizemos com desenhos de modo a permitir uma avaliação de diferentes protagonistas do ambiente escolar. 


\section{Referências}

ALVES-MAZZOTTI, A. J. Do trabalho à rua: uma análise das representações sociais produzidas por meninos trabalhadores e meninos de rua. In: JORNADAS DE PESQUISADORES EM CIÊNCIAS HUMANAS, 4., 1997, Rio de Janeiro. Tecendo saberes. Rio de Janeiro: CFCH, UFRJ: Fundação Universitária José Bonifácio, 2000. 1 CD-ROM.

ANZIEU, D. Os métodos projetivos. Rio de Janeiro: Campus, 1979.

BARDIN, L. L'analyse de contenu. Paris: Presses Universitaires de France, 1991.

BARTHES, R. Mythologies. Paris: Éditions du Seuil, 1957.

BOURDIEU, P. La distinction. Paris: Editions de Minuit, 1979.

. Reprodução cultural e reprodução social. In: MICELI, S. (Org.). A economia das trocas simbólicas. São Paulo: Perspectiva, 1987.

COSTA, W. A. A.; OLIVERA, A. M. A construção social do conceito de Bom Professor. Tradução por Pedro Humberto Faria Campos. In: MOREIRA, A. S. P.; OLIVEIRA, D. C. (Org.). Estudos interdisciplinares de representações sociais. Goiânia: $A B, 1998$.

DE MĖREDIEU, F. Le dessin d'enfant. Paris: Blusson, 1990.

DE ROSA, A. S. The social representations of mental illness in children and adults. In: DOISE, W.; MOSCOVICI, S. (Org.). Current issues in social psychology. Cambridge: CUP, 1987.

EMLER, N.; OHANA, J.; MOSCOVICI, S. Children's beliefs about institutional roles: a cross-national study of representations of the teacher's role, Br. J. Educ. Psychol., London, n. 57, p. 26-37, 1987.

ESQUIVEL, J. M. As representações da escola e o desempenho escolar. 1997. Trabalho de Conclusão de Curso (Graduação em Pedagogia) - Faculdade de Educação, Universidade Federal do Rio de Janeiro, Rio de Janeiro, 1997.

FISCHER, G. N. Psychosociologie de l'espace. Paris: Presses Universitaires de France, 1980. . Psychologie sociale de l'environnement. Toulouse: Editions Privat, 1989. 
GALLI, I.; NIGRO, G. The social representation of radioactivity among Italian children. Social Science Information, v. 26, n. 3, p. 535-549, 1987.

GILLY, M. Psicosociologia de la educación. In: MOSCOVICl, S. (Org.). Psicologia social. Barcelona: Paidós, 1984. v. 2.

GRAUMANN, C. F. Le concept d'appropriation (Aneignung) et les modes d'appropriation de l'space, [S. I.], 1979. Mimeografado.

GRAUMANN, C. F.; KRUSE, L. Masas, muchedumbres y densidad. In: Moscovici, S. (Org.). Psicologia social. Barcelona: Paidós, 1984.

GUMP, P. V. School and classroom environments. In: STOKOLS, D.; ALTMAN, I. (Ed.). Handbook of environmental psychology. New York: John Wiley \& Sons, 1987.

GOFFMAN, E. Asyluns. Garden City, Nova York: Anchor, 1961.

JODELET, D. Le corps, la persone et autrui. In: MOSCOVICl, S. (Ed.). Psychologie sociale dês relations à autrui. Paris: Nathan, 1994.

JOLY, M. Introduction à l'analyse de l'image. Paris: Nathan, 1993.

LE BON, G. La psychologie des foules. Paris: Presses Universitaires de France, 1963.

LEWIN, K. Resolving social conflicts. Nova York: Harper \& Row, 1948.

LOURENÇO, M. A. Representações socioculturais e a designação metacognitiva em aprendizagem bilíngue (línguas tchuwabo e portuguesa): implicações para o ensino fundamental em Moçambique. 2005. Tese (Doutorado em Psicologia) Programa de Pós-graduação em Psicologia, Universidade Federal do Rio de Janeiro, Rio de Janeiro, 2005.

MARSH, P.; ROSSER, F.; HARRÉ, R. The rules of disorder. London: Routledge and Kegan Paul, 1978.

MILGRAM, S. Cities as social representations. In: FARR, R. M.; MOSCOVICl, S. (Org.). Social representations. London: Cambridge University Press/Editions de la Maison des Sciences de l'Homme, 1984. 
MOSCOVICl, S. La Psychanalyse, son image et son public. Paris: Presses Universitaires de France, 1976. Tradução brasileira: A representação social da psicanálise. Rio de Janeiro: Zahar, 1978.

РАТТО, M. H. S. Da psicologia do 'desprivilegiado' a psicologia do oprimido. In: . (Org.). Psicologia escolar. São Paulo: T. A. Queiroz, 1980.

SALES, L. C. Prédios escolares: representações sociais das escolas. Revista de Ciências Humanas, Florianópolis, SC, p. 333-342, 2002.

. O valor simbólico do prédio escolar. Teresina: EDUFPI, 2000.

SOMMER, R. Personal space: the behavioral basis of design. Englewood Cliffs, NJ: Prentice Hall, 1969.

TAJFEL, H. Human groups and social categories. Cambridge: Cambridge University Press, 1981.

TEDESCO, J. C. A dinamização do sistema educativo. In: Gadotti, M. (Org.). História das idéias pedagógicas. São Paulo: Ática, 1996.

VAYER, P.; DUVAL, A.; RONCIN, C. Una ecología de la escuela. Barcelona: Paidós, 1993.

VIGOSKII, L. S. La imaginación y el arte em la infância: ensayo psicológico. Madri: Ediciones Akal, 1996.

WEBER, S.; MITCHELL, C. Using drawings to interrogate professional identity and the popular culture of teaching. In: GOODSON, I. F.; HARGREAVES, A. (Ed.). Teacher's professional lives. London: Falmer Press, 1996.

Recebido em: 12/02/2009

Aceito para publicação em: 08/10/2009 